\title{
A Geometric Contour Framework with Vector Field Support
}

\author{
Zhenglong Li, Qingshan Liu, and Hanqing Lu \\ National Laboratory of Pattern Recognition \\ Automation of Institute, Chinese Academy of Sciences \\ P.O. Box 2728, Beijing, Zip Code: 100080 \\ \{zlli, qsliu, luhq\}@nlpr.ia.ac.cn
}

\begin{abstract}
In this paper, we propose a new geometric contour framework with support of specified vector field. First we define three criteria for selection of vector field in geometric model. According to the criteria, EdgeFlow, a powerful segmentation tool, is selected to generate desirable initial vector field. In order to overcome the drawbacks of conventional geometric models, multi-source external forces, such as from texture and multi-spectra, are integrated to provide the ability for segmenting the texture-rich and complex scene images. Instead of common smoothing pre-processing to denoise and suppress possible spurious edges, the more advanced complex diffusion filters are adopted in our algorithm, which result in the piecewise filtered image to help detect those sharp transition regions. We test our model on the Berkeley Segmentation Database, and the experimental results are promising.
\end{abstract}

\section{Introduction}

In recent decades, Curve Evolution methods have been extensively exploited in computer vision society due to their potential applications in object contour extraction/object segmentation, motion estimation\&tracking, et al. According to the expressions of mathematical models, they are roughly divided into two classes: Parametric Curve Model and Geometric Curve Model/Non-Parametric Curve Model. The representative of the parametric curve models is Snakes [1]: it drives a parameterized curve by image-oriented force to positions of interests. And the geometric curve model is based on the theory of Level Set [2] which can deal with topological changes during evolution process without any additional intervention. Contrast with the constraints of snakes, geometric curve models have two advantages: 1) They can deal with the case that the number of objects in scene is unknown; 2) No additional re-parameterization maneuver is needed during curve evolution.

The original geometric curve models were introduced by Malladi et al [3] and Caselles et al [4] respectively. The ideas of them utilize the grayscale/single spectral information as the external force to drive curve to positions of interests. Though these algorithms achieve successes to some extent, there also exist some limitations. First, most them use simple grayscale gradient, so that they merely 
tackle "simple" images, i.e. synthesized images or images with clear edge cues. As for the rich textural context or presence of clutters, they fail to converge to positions of interests. Second, usually only single source image-oriented information is integrated into model, whereas other useful and important information such as color, texture do not get their places in curve evolution. For those models using single cue as driven force, it is difficult to get reliable external force in those region of indistinct single cue. Some researchers $[5,6]$ have noted these situations and proposed solutions for textural images. Recently Xie [7] gives an interesting attempt to integrate information of region-based segmentation into model.

In this paper, we propose a novel curve evolution framework, which can handle segmentation in some relatively texture-rich and/or complex context images. We first define three criteria for customized vector field. Based on these criteria, an effective algorithm, i.e., EdgeFlow [8], is selected to generate desirable initial vector field. In order to deal with the drawbacks of the conventional geometric models, multi-source external forces, such as forces from texture and multi-spectra, are integrated to provide the ability to segment the texture-rich and complex scene images. We adopt a so-called "generalized gradient" method to extract change information in multi-spectral/vector images (we treat color images and Gabor filtered multi-channel images as multi-spectral image in a sense of signal processing). "Generalized gradient" has been proven an effective and simple method in multi-spectral image analysis [9]. Through proper diffuse processing of initial vector field, the desirable vector fields are achieved. Instead of common smoothing pre-processing to denoise and suppress possible spurious edges, the more advanced complex diffusion filters are adopted in our algorithm, which result in the piecewise filtered image to help detect those sharp transition regions in image. We test our model on the Berkeley Segmentation Database and the experimental results show effectiveness of our method.

The proposed method is different from [6]. The difference is that they try to divide vector field of EdgeFlow into the irrational and solenoidal vector fields. By solving a Poisson PDE, they get an edge function $V$, and then edge flow and edge function are integrated into geometric contour framework. But this method directly relies on pre-processing of EdgeFlow. Instead of depending on any single existing vector field generation scheme, we propose the general criteria for customizing vector field in curve evolution. Any vector field that satisfies those criteria can be taken into the geometric curve model. Moreover, multi-source cues can be integrated into the model to get reliable result. There also exist the differences between our method and [7]. In [7], they use pre-segmentation by Mean Shift to derive region-related driven force to overcome edge leakage and then GVF to extend capture range. And for pre-segmentation map, direct GVF diffuse process cannot guarantee a regular vector field under clutter presegmentation result or complex scene. This method still only segment simple images, and fails in dealing with textural or complex scene images. The proposed framework combines diffusion enhancement and vector field generation method to overcome these disadvantages. 


\section{A Brief Review of Geometric Contour Model}

Geometric active contour model is based on the curve evolution theory. Usually it needs initial curve $C_{o}$ to be specified beforehand, and it uses the external force derived from image to drive curve to positions of interests. The process of curve evolution is described by the coupled partial differential equations (PDEs). Compared with conventional parametric numerical methods, Level Set method [2] has the advantages of adaption to topological changes, i.e. curves merging and/or splitting in an automatic manner without any additional intervention, while parametric contour model cannot easily handle those situations. Some works based on parametric contour model try to deal with topological changes, but they bring with a huge computation complexity [10].

The geometric contour model is introduced respectively by Malladi et al [3] and Caselles et al [4]. In [3], the following PDE is used as

$$
C_{t}=g(|\nabla I|)(\kappa+c) \boldsymbol{N},
$$

where $C$ is a 2-D closed contour, $g(\cdot):[0, \infty] \mapsto[1,0]$ is a monotonic decreasing function, $\kappa$ and $c$ are curvature and constant speed item respectively, and $\boldsymbol{N}$ is the unit normal vector of curve with inward direction. It is obvious that $g(\cdot)$ will decrease quickly to zero when contour is near edge, so the advancing curve will be stalled for total speed $C_{t}$ approximating to zero. In (1) constant $c$ is an artificial balloon-like force [11] to drive curve in those feature-lacking region to contract/expand to object boundary. However this model has a defect that curve cannot come back if the curve steps beyond object boundary.

In [4], an improved model is proposed as follows

$$
\left.C_{t}=g(|\nabla I|)(\kappa+c) \boldsymbol{N}-(\nabla g(|\nabla I|)) \cdot \boldsymbol{N}\right) \boldsymbol{N},
$$

in which an additional item is introduced in right hand side of (2) compared with (1). This new item offers curve the ability to come back when going beyond object boundary. But the capture range for pure gradient-deduced vector force, $(\nabla g(|I|)) \cdot \boldsymbol{N}) \boldsymbol{N}$, is very limited, because it is determined by used gradient operator, e.g. for the most used derivative of Gaussian operator, capture range is affected by $\sigma$.

Because stopping items in (1) and (2) is too weak to counter geometric force to get balance, in the case that image-derived gradient is small, both model (1) and (2) fails to handle weak edges.

\section{Our Works}

\subsection{Criteria for Vector Field}

From a viewpoint of dynamics, there are two kinds of strategies to stop the motion of the curve. One is considering scalar function $g(\cdot)$ as edge indicator such that $g(\cdot)$ will attenuate to zero quickly, and therefore decrease the total 
speed in (1) and first speed item in (2) to zero, when encountering high change rate of local feature, e.g. gradient as the most usual choice. The other is to add vector force $\nabla g(\cdot)$ to balance the image-oriented force at positions where vector flows bump into. The convergence is achieved only when whole system gets to equilibrium between the geometric constraint and the image-oriented forces. Both strategies employed in (1) and in (2) cannot handle weak edges for noise, illumination, and albedo et al. Only relying on $g(\cdot)$ or gradient flow from gradient operator is not enough to stop curve moving over those weak edges and/or scarce feature regions.

In this paper, we design a novel framework to deal with problems that conventional models cannot solve, such as edge leakage and applications in texture-rich and/or complex scene. Instead of designing complicated $g(\cdot)$ or considering to design new external force, we consider that the drawbacks, i.e. weak edge leakage, capture range, and textural image segmentation, et al, can be rescued by a vector flow field which satisfies some criteria, from a viewpoint of separation of underlying vector field from the geometric contour model. In the following, we give the definition of these criteria.

Many studies have contributed to the solution of the problems in curve evolution. In [11], Cohen et al mentioned only using direction information of gradient vector as external driven force by which head-to-head vector flows between edge are constructed, and this scheme can make contour converge to object boundary. The idea of Xu et al [12] and Yuan [13] are similar. They all deduce vector field from edge map, for those vectors in the regions far from edges, and all can be traced back to edges. From the viewpoint of field theory, we can regard those methods as generating the conservative field, in which every point of curve acts like a free particle effected by the conservative field force and geometric constraints.

Here, we propose three new criteria for vector field in geometric model. 1) $D i$ rection Criterion. Between edges, vector flow should be head-to-head pointing toward each other; 2) Energy Criterion. Vectors in the vicinity of edges should possess dominant energy; 3) Attraction Criterion. For any region far from edges, there should exist vector flow pointing toward edge or the vector tracing back toward edge. We give some brief explanations on these three criteria as follows.

The purpose of criterion 1) is to stop moving curve and accurately locating edge positions. Assuming some part of deforming curve is across the edge while countering constant balloon-like force and geometric constraint, it is possible that parts of curve moves beyond edges. Thus, we require the head-to-head vector flow to draw curve back when curve is beyond edge location. Moreover, if we remove balloon-like force to allow curve under effect of only vector flow and geometric constraint, we will get an improvement of final convergence to get accurate positioning to edge. But it brings with a problem that contour model will become sensitive to noise points and/or meaningless blobs. The energy criterion we define is of two-fold purposes. First, around edge the attraction should reach extrema to trap deforming contour. Second, dominant energy can accelerate convergence process near edges, contrast with the method of only 
using directional information [11]. Since initial contour may be not prescribed exactly in vicinity of object. In those regions far from edge, the deforming curve is wished to be still attracted by edge, while balloon-like force should be set as small as possible for the consideration of edge leakage. That's the reason why we contrive attraction criteria.

One aim of our framework is that any vector field satisfying these three criteria above can be integrated into the geometric model. To demonstrate the effectiveness of the criteria, we choose EdgeFlow [8] as the candidate vector field, although edge flow does not fully satisfy the criteria we define above. But we find that if we diffuse edge flow vectors with GVF method [12], the modified vector field can satisfy well all the three criteria.

EdgeFlow is a powerful tool for boundary detection and image segmentation. It cannot only incorporate single grayscale information, but also other information such as the color, Gabor phase and et al (for details, cf. [8]). This method was tested on a large number of natural images, and gave the good performance. Fig. 1(b) shows the edge flows in the white square of the left subfigure (a).

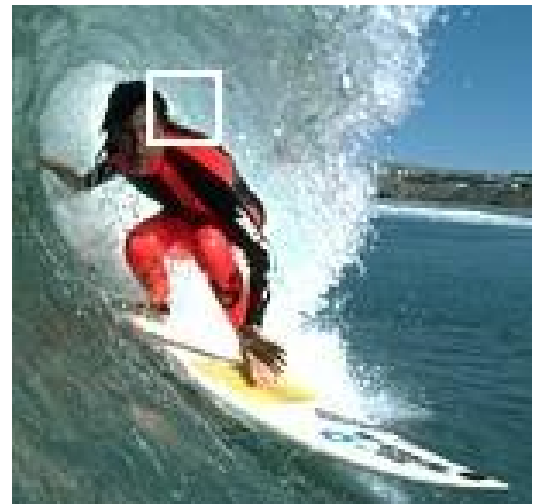

(a)

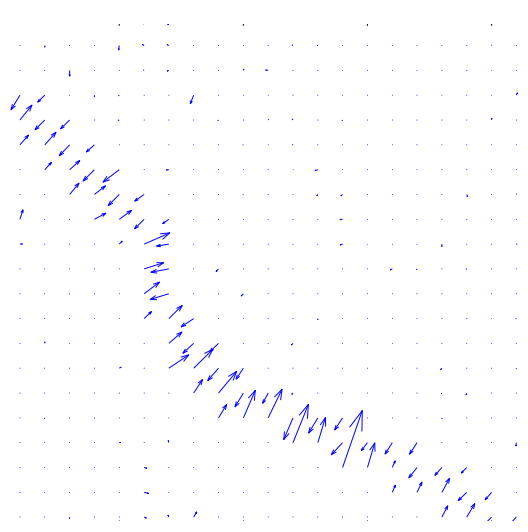

(b)

Fig. 1. Vector field formed by EdgeFlow method. (a) The part of original image. (b) Magnified vector field by EdgeFlow in white square region of (a).

\subsection{Complex Diffusion and Generalized Gradient}

In order to suppress spurious edges and reduce effect of noise, smoothing operation is an usual pre-processing method before any operation extracting useful information in images. The widely used multi-scale smoothing scheme is Gaussian pyramid. But this scheme will bring with the implementation difficulties for curve evolution across different scales [14] and lose accurate edge locating ability. To deal with these problems, a more advanced diffusion process called 
complex diffusion process is adopted in this paper. The complex diffusion process produces filtered images with piecewise properties.

For multi-spectral/multi-value images, simply algebraic combination of each channel's response cannot give a fine description on change rate of local features. We use "generalized gradient" [15] to describe local feature changes in multispectral images, for it is a robust and effective method ever proven.

Complex Diffusion In low level vision tasks, filtering is often used to reduce the influence of noise and meaningless clutter. For the images with rich texture or complex scene, conventional filtering cannot fulfil this task. Scale-space approach is a proven useful technique in image processing. And it is known that PeronaMallik diffusion is an adaptive diffusion process to the different scale, although this scheme cannot deal with texture-rich image for its sensibility to variation of gradient. Shock filters [16] suffer the same shortcoming. We use the complex diffusion [17] to do smoothing inside homogeneous region while enhancing edge. We use this more advanced PDE-based diffusion process to smooth inside region while sharpening edge. The complex shock filter used in this paper is

$$
I_{t}=-\frac{2}{\pi} \arctan \left(a \operatorname{Im}\left(\frac{I}{\theta}\right)\right)|\nabla I|+\lambda I_{\eta \eta}+\tilde{\lambda} I_{\xi \xi},
$$

where $\lambda=r e^{i \theta}$, and $\tilde{\lambda}$ is a real scalar value (for more details, cf. $[16,17]$ ).

The complex diffused image is composed of real part and imaginary part, i.e. the value of each point is a complex value. The real part and imaginary part of complex filters play the different roles in diffusion process. The real part of complex filters behaves like an adaptive smoothing operator, while the imaginary part of complex filters is similar to a simple second derivative operator, but offers more advantages than it.

For multi-spectral images, e.g. color images and Gabor-filtered images, we apply this complex shock filter to each sub-band image respectively to denoise and enhance its edges, and then "generalized gradient" will be extracted from sub-band images to represent the change rate cross all spectra. Fig. 2 gives a complex diffusion processed image. It is obvious to see that edges are enhanced and inside region is well smoothed. In fact, this diffusion process can be seen as a reconstruction process of piecewise image.

Generalized Gradient For a multi-spectral/multi-value image $I$ with $m$ channels, we can regard the imaging process as a function $f: \mathbb{R}^{2} \mapsto \mathbb{R}^{m}$. A point in the image is a m-vector. Now, we use the approach named "generalized gradient" to detect changes in local feature of $\mathbb{R}^{m}$.

Consider two points $P$ and $Q$ in $\mathbb{R}^{m}$, i.e. two m-vectors respectively. Difference between them is $\Delta \mathbf{f}=\mathbf{f}(P)-\mathbf{f}(Q)$. When the distance between $P$ and $Q$ is infinitesimal, i.e. $d(P, Q)$ tending to zero, we can get its squared norm in matrix notation $[15]$

$$
\mathrm{d} \mathbf{f}^{2}=\left[\begin{array}{l}
\mathrm{d} x_{1} \\
\mathrm{~d} x_{2}
\end{array}\right]^{T}\left[\begin{array}{ll}
g_{11} & g_{12} \\
g_{21} & g_{22}
\end{array}\right]\left[\begin{array}{l}
\mathrm{d} x_{1} \\
\mathrm{~d} x_{2}
\end{array}\right],
$$




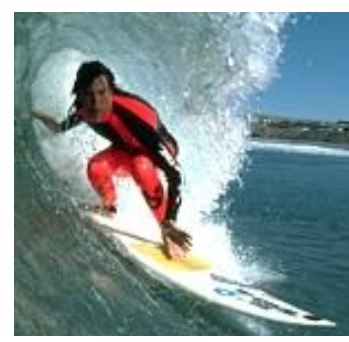

(a)

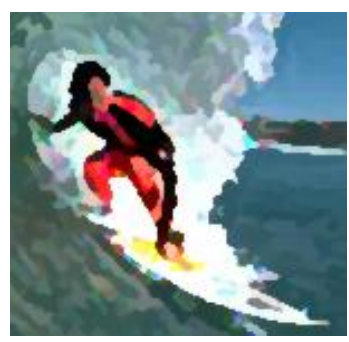

(b)

Fig. 2. Result by complex shock filter. (a) The original image. (b) The filtered image with piecewise property.

where

$$
g_{i j}=\sum_{k=1}^{m} \frac{\partial f_{k}}{\partial x_{i}} \frac{\partial f_{k}}{\partial x_{j}} .
$$

Note that $d \mathbf{f}^{2}$ indicates the change rate along direction $P-Q$. In the directions of two eigenvector of matrix $\left[g_{i j}\right]$, the equation (4) gets two extrema, i.e. the maximum value and the minimum value respectively. Here $\left[g_{i j}\right]$ is a $4 \times 42$-D matrix, and its eigenvalues are given as follows

$$
\lambda_{ \pm}=\frac{g_{11}+g_{22} \pm \sqrt{\left(g_{11}-g_{22}\right)^{2}+4 g_{12}^{2}}}{2} .
$$

and the corresponding eigenvectors are

$$
l_{ \pm}=\left(\cos \theta_{ \pm}, \sin \theta_{ \pm}\right)^{T}
$$

where

$$
\begin{aligned}
& \theta_{+}=\frac{1}{2} \arctan \frac{2 g_{12}}{g_{11}-g_{22}}+k \pi, \quad \text { and } \\
& \theta_{-}=\theta_{+} \pm \frac{\pi}{2} .
\end{aligned}
$$

When $m$, the number of sub-bands, is equal to $1, \lambda$ is equivalent to gradient in the case of 2-D single-value image. Thus, it is called "generalized gradient".

Replacing $\nabla I$ of gradient with $\lambda_{+}$, we integrate components of multi-spectral image information into the geometric model. The change rate in any multispectral image can be described by this method. In our experiments, only Gaborfiltered images and color RGB images are used.

In order to modify vector field generated by EdgeFlow for the proposed criteria, we use GVF [12] to diffuse vector field of edge flow. The idea of GVF is to diffuse edge map to get vector field for extending capture range. The GVF's diffusion equations are two coupled PDEs:

$$
\mu \nabla^{2} u-\left(u-I_{x}\right)\left(I_{x}^{2}+I_{y}^{2}\right)=0,
$$




$$
\mu \nabla^{2} v-\left(v-I_{y}\right)\left(I_{x}^{2}+I_{y}^{2}\right)=0 .
$$

We modify the edge items $\left(I_{x}^{2}+I_{y}^{2}\right)$ in $(7)$ as $\lambda_{+}$in sub-band images and set initial condition of (7) as vector field from EdgeFlow:

$$
\begin{aligned}
& \mu \nabla^{2} u-\left(u-E_{x}\right) \lambda_{+}=0, \\
& \mu \nabla^{2} v-\left(v-E_{y}\right) \lambda_{+}=0,
\end{aligned}
$$

where $E_{x}, E_{y}$ are x, y component of vector in EdgeFlow.

With integration of EdgeFlow, "generalized gradient", and complex diffusion, we give our geometric model with combination of multi-source external forces as

$$
C_{t}(p)=\left(\alpha g(|\nabla I|)(\kappa+c)-\beta \nabla g\left(\lambda_{+}\right) \cdot \boldsymbol{N}+\gamma g(|\boldsymbol{F}|) \frac{\boldsymbol{F}}{|\boldsymbol{F}|} \cdot \boldsymbol{N}\right) \boldsymbol{N},
$$

where $\boldsymbol{F}=(u, v)^{T}$ is the vector field from (8), and $\alpha, \beta, \gamma$ are weight coefficients respectively.

In (9), we integrate the multi-source external forces and the customized vector field into our model. The second item $\nabla g\left(\lambda_{+}\right) \cdot \boldsymbol{N}$ in right hand side of (9) incorporates the generalized gradient to utilize multi-spectral information. And in the last item $\boldsymbol{F}$ is the vector field force derived from edge flow. The customized $\boldsymbol{F}$ offers model the ability to segment the texture-rich and/or complex images. Furthermore, with a strategy of multi-source external forces and customized vector field, the proposed model can overcome the drawbacks of conventional models mentioned in previous section.

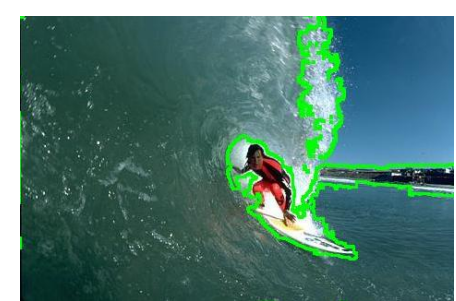

(a)

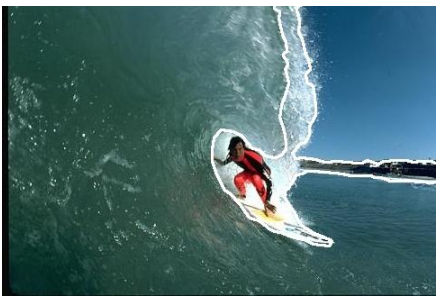

(b)

Fig. 3. Segmentation results by EdgeFlow and our method, respectively. (a) The result of original EdgeFlow. (b) The corresponding result by our method.

\section{Experiments}

We test our model on the Berkeley Segmentation Database. Any vector field which satisfies the proposed criteria can be integrated into (9). In test, we choose EdgeFlow to generate initial vector field (for implementation issues, cf. [8]). Then 
by (8), we modify the original edge flow vector field to conform to our criteria, so the desirable vector field has been constructed. Moreover, to describe the change of local feature in multi-spectral image, we use "generalized gradient". The initial curves are set manually and a post-process of region merging is adopted in the test.

See Fig. 3(a), it is the segmentation result by the original EdgeFlow method [8]. In the middle of the image, we can find the difference between white water wave and surfing man cannot be well segmented by EdgeFlow. And in the right upper part of image, the white water wave has the trend to be over-segmented. Fig. 3(b) shows the corresponding result by our method, and it can be seen that the proposed method gives a finer segmentation result.

Fig. 4 shows some other examples of segmentation with our method. These examples have rich texture and complex scene content. Our method still gives the interesting segmentation results. Although some little defects still exist in segmentation results, it must be pointed out that segmenting image with texturerich content and/or complex scene is a very challenging work.
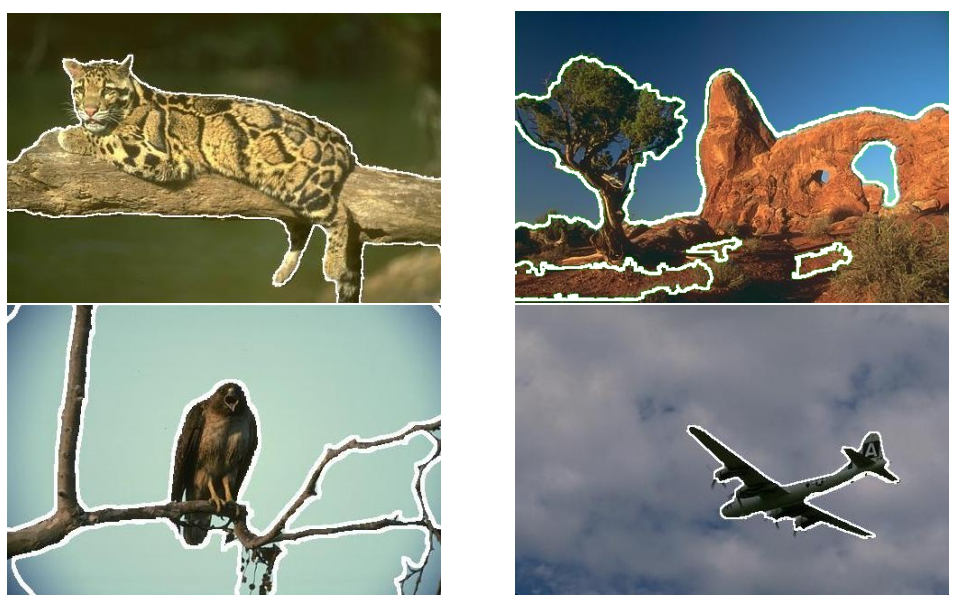

Fig. 4. Some other experimental results by our method.

\section{Conclusion}

We propose a novel geometric contour model with support of customized vector field. Multi-source external forces are integrated into model to give high reliable performance. Contrast with conventional model which can only deal with simple image, our model can be applied to relatively complex and textural images. We do tests on Berkeley Segmentation Database and the result is promising. 


\section{Acknowledgment}

We would like to acknowledge support from Natural Sciences Foundation of China under grant No. 60135020, 60475010 and 60121302.

\section{References}

1. Kass, M., Witkin, A., Terzopoulos, D.: Snakes: Active contour models. Int. Jnl. of Comp. Vis. 1 (1988) 321-331

2. Osher, S., Sethian, J.A.: Fronts propagating with curvature dependent speed: Algorithms based on Hamilton-Jacobi formulations. Journal of Computational Physics 79 (1988)

3. Malladi, R., Sethian, J.A., Vemuri, B.C.: Shape modeling with front propagation: A level set approach. IEEE Trans. Pattern Anal. Machine Intell. 17 (1995) 158-175

4. Caselles, V., Kimmel, R., Sapiro, G.: Geodesic active contours. Int. Jnl. of Comp. Vis. 22 (1997) 61-79

5. Paragios, N., Deriche, R.: Geodesic active regions and level set methods for supervised texture segmentation. Int. Jnl. of Comp. Vis. 46 (2002) 223-247

6. Sumengen, B., Manjunath, B.S., Kenney, C.: Image segmentation using curve evolution and flow fields. In: IEEE Int. Conf. on Image Processing. (2002)

7. Xie, X., Mirmehdi, M.: RAGS: Region-aided geometric snake. IEEE Trans. Image Processing 13 (2004) 640-652

8. Ma, W., Manjunath, B.S.: EdgeFlow: A technique for boundary detection and image segmentation. IEEE Trans. Image Processing 9 (2000) 1375-1388

9. Cumani, A.: Edge detection in multispectral images. Computer Vision, Graphics, and Image Processing: Graphical Models and Image Processing 53 (1991) 40-51

10. McInerney, T., Terzopoulos, D.: Topologically adaptable snakes. In: IEEE Int. Conf. on Comp. Vis., Cambridge, MA (1995) 840-845

11. Cohen, L.D., Cohen, I.: Finite element methods for active contour models and balloons for 2-D and 3-D images. IEEE Trans. Pattern Anal. Machine Intell. 15 (1993) 1131-1147

12. Xu, C., Prince, J.L.: Snakes, shapes, and gradient vector flow. IEEE Trans. Image Processing 7 (1998) 359-369

13. Yuan, D., Lu, S.: Simulated static electric field(SSEF) snakes for deformable models. In: Int. Conf. on Patt. Recog. Volume 1. (2002) 83-86

14. Leroy, B., Herlin, I.L., Cohen, L.D.: Multi-resolution algorithms for active contour models. In: Proc. of the 12th Intl. Conf. on Analysis and Optimization of Systems Images, Wavelets and PDE'S, Rocquencourt, France (1996)

15. Zenzo, S.D.: A note on the gradient of a multi-image. 33 (1986) 116-125

16. Alvarez, L., Lions, P.L., Morel, J.M.: Image selective smoothing and edge detection by nonlinear diffusion. II. SIAM J. Numer. Anal. 29 (1992) 845-866

17. Gilboa, G.: Image enhancement and denoising by complex diffusion processes. IEEE Trans. Pattern Anal. Machine Intell. 26 (2004) 1020-1036 